MATHEMATICS OF COMPUTATION

Volume 71, Number 239, Pages 1169-1188

S 0025-5718(01)01338-2

Article electronically published on October 25, 2001

\title{
APPROXIMATION OF THE HILBERT TRANSFORM ON THE REAL LINE USING HERMITE ZEROS
}

\author{
M. C. DE BONIS, B. DELLA VECCHIA, AND G. MASTROIANNI
}

\begin{abstract}
The authors study the Hilbert Transform on the real line. They introduce some polynomial approximations and some algorithms for its numerical evaluation. Error estimates in uniform norm are given.
\end{abstract}

\section{INTRODUCTION}

Let us consider the integral

$$
H(G, t):=\int_{\mathbb{R}} \frac{G(x)}{x-t} d x=\lim _{\epsilon \rightarrow 0^{+}} \int_{|x-t| \geq \epsilon} \frac{G(x)}{x-t} d x,
$$

where $t \in \mathbb{R}$. If we assume that the limit on the right-hand side exists, then $H$ is called Hilbert Transform. It appears in several mathematical problems and, essentially, it is the main part of the singular integral equations on $\mathbb{R}[28$. Therefore approximations and numerical evaluations of $H(G, t)$ are of great interest.

It is well known that $H$ is a bounded map in the $L^{p}$ spaces, $1<p<\infty$, and that it is usually considered in the $L^{2}$ spaces where $\pi^{-1} H$ is an isometric isomorphism [1]. In the space of the continuous functions equipped with the uniform metric, the Hilbert Transform is an unbounded operator.

However, if we assume that the Dini type condition

$$
\int_{0}^{1} \frac{\omega(G, u)}{u} d u<\infty
$$

holds, where $\omega$ is the ordinary modulus of smoothness on $\mathbb{R}$, then $H(G, t)$ is a continuous function on $\mathbb{R}[14$ Theorem 2.24, p. 218].

In the last decade several papers have dealt with the numerical approximation of the Hilbert Transform in the case of bounded intervals and the reader can refer to [4], [5], 6], 7], [13 [18, 20], [25] and [26]. The algorithms proposed in these papers are mainly two: Gauss-type quadrature rules and product quadrature rules. The first ones subtract the singularity and apply an ordinary Gauss quadrature rule using an additional algorithm to control the term of the quadrature sum containing

Received by the editor April 9, 1998 and, in revised form, December 8, 1999, May 12, 2000, and August 18, 2000.

2000 Mathematics Subject Classification. Primary 65D30, 41A05.

Key words and phrases. Hilbert Transform, orthonormal polynomials, Gaussian quadrature rules, product quadrature rules.

This work was supported by M.U.R.S.T. (ex. 40\%). 
the knot closest to the singularity [4, [13, 27]. The second ones consist in substituting the integrand function by its interpolating polynomial having Lebesgue constants of order $\log m$.

On the other hand the literature concerning the numerical integration on unbounded intervals is by far poorer than the one on bounded intervals. For instance, in the case of integral transforms with continuous or weakly singular kernels, the convergence of some product quadrature rules has been proved in [19], 29], [33. and [34. Estimates of the quadrature error have been recently proved in [21], 222, 23] and [27]. The case of the Hilbert Transform has been considered very little and the reader can consult [3], [9, [15], 16], [35], 36], [37, 38] and the references given there.

In particular in [16 the authors assume that the function $G$ is analytic in the strip $\{\mathbb{C}:|\Im z|<d\}$, in which case they show that the series

$$
2 \sum_{\substack{\nu=-\infty \\ \nu \neq \text { even }}}^{\infty} \frac{G(t+\nu h)}{\nu},
$$

converges to $H(G, t)$ at the rate $\mathcal{O}\left(e^{-\frac{\pi d}{h}}\right)$ as $h \rightarrow 0$. Successively they approximate $H(G, t)$ by a partial sum of the above series. Obviously the error depends on the decay to $\pm \infty$ of the function $G$.

In [3] the author replaces the above series with the following one

$$
\sum_{k=-\infty}^{\infty} \frac{G\left(t+k h+\frac{h}{2}\right)}{k+\frac{1}{2}}
$$

for a suitable choice of the step $h \rightarrow 0$. Proceeding as in [16] and making suitable transformations (Sinc Method), this procedure can be used for piece-wise analytic functions (see [35], [36], [37, [38]) which frequently appear in the applications.

In this paper we propose to approximate $H(G, t)$ by using the zeros of Hermite polynomials. More precisely, we write $G(x)=\left[G(x) e^{(p x)^{2}}\right] e^{-(p x)^{2}}:=f(x) e^{-(p x)^{2}}$, $p \neq 0$, and we assume the function $f$ belonging to suitable Sobolev spaces and such that, for $r \geq 1$ and $|x|>x_{0}>0,\left|f^{(r)}(x) e^{-(p x)^{2}}\right|$ decays to $\pm \infty$ algebraically. Since the zeros of the polynomial $H_{m}(x)$ are in the interval $[-p \sqrt{2 m}, p \sqrt{2 m}]$, taking into account the decay of $\left|f^{(r)}(x) e^{-(p x)^{2}}\right|$, the computation of $H(G, t)$ is reduced to the computation of an analogous integral on a finite interval (depending on $m$ ). This circumstance allows us to use the procedures proposed in [4], [5], 6], [7, [13], [18, [20], 25] and [26].

In Section 3 we propose a Gauss-type quadrature rule and a product quadrature rule.

The Gauss-type quadrature rule is useful because it has the computational cost of an ordinary Gaussian formula and it controls the term of the formula containing the zero closest to the singularity. This circumstance requires additional information on the distance of the zeros of two consecutive Hermite polynomials (see Lemma 2.11).

The product quadrature rules have not been studied till now, because interpolation processes having Lebesgue constants (in $W_{0}^{\infty}$ ) of order $\log m$ were not available. Using an idea by J. Szabados [39, we construct a simple interpolating Lagrange polynomial and we prove that it is optimal in $W_{0}^{\infty}$ (see Theorem[2.2). Subsequently we approximate $H(G, t)$ by replacing $f$ with the above-mentioned polynomial. We 
obtain a formula having greater computational cost than the one of the Gauss-type formula, but it proves to be more useful in collocation methods for solving singular integral equations on $\mathbb{R}$. In Theorem $[3.2$, we establish convergence conditions for this procedure. In the same section we propose a simple algorithm when the parameter $t$ is "large".

Extensive numerical testing and comparisons with other procedures have been performed and all the results confirm our theoretical estimates. Finally, in Section 5 we show some significant examples.

\section{Preliminary Results}

Functional spaces. In the following $\mathcal{C}$ denotes a positive constant which may assume different values in different formulas. In the sequel, $\mathcal{C} \neq \mathcal{C}(a, b, \ldots)$ means that $\mathcal{C}$ is independent of $a, b, \ldots$. Moreover we write $A \sim B$, for $A, B>0$, iff there exist two positive constants $M_{1}, M_{2}$, independent of $A$ and $B$, such that

$$
M_{1} \leq\left(\frac{A}{B}\right)^{ \pm 1} \leq M_{2}
$$

As we have already announced in the introduction, the main aim of this paper is the numerical approximation of the integral

$$
\int_{\mathbb{R}} \frac{G(x)}{x-t} d x, \quad t \in \mathbb{R},
$$

by using Hermite zeros. In order to do this, we write the function $G$ as $G(x)=$ $\left[G(x) e^{(p x)^{2}}\right] e^{-(p x)^{2}}:=f(x) e^{-(p x)^{2}}, \quad p \neq 0$, and we successively construct the weighted polynomial approximation of the function $f$. To this end the following preliminary results are useful.

With respect to $w_{p}(x)=e^{-(p x)^{2}}$, let us consider the following set of locally continuous functions

$$
W_{0}^{\infty}:=W_{0}^{\infty}\left(w_{p}\right)=\left\{f \in C_{\mathrm{LOC}}^{0}(\mathbb{R}): \lim _{|x| \rightarrow \infty} w_{p}(x) f(x)=0\right\}
$$

which, equipped with the norm

$$
\|f\|_{W_{0}^{\infty}}:=\left\|f w_{p}\right\|_{\infty}=\max _{\mathbb{R}}\left|f(x) w_{p}(x)\right|,
$$

is a Banach space. For smoother functions we consider the usual Sobolev space $W_{r}^{\infty}, r \geq 1$, given by

$$
W_{r}^{\infty}:=\left\{f \in W_{0}^{\infty}:\left\|f^{(r)} w_{p}\right\|_{\infty}<\infty\right\}
$$

with the norm

$$
\|f\|_{W_{r}^{\infty}}:=\left\|f w_{p}\right\|_{\infty}+\left\|f^{(r)} w_{p}\right\|_{\infty} .
$$

For all $f \in W_{0}^{\infty}$ we define the following weighted modulus of smoothness

$$
\Omega^{k}(f, t)_{w_{p}, \infty}:=\sup _{0<h \leq t|x| \leq \frac{1}{h}} \max _{h}\left|\Delta_{h}^{k} f(x)\right| w_{p}(x),
$$

where $k \geq 1$ and

$$
\Delta_{h}^{k} f(x):=\sum_{i=0}^{k}(-1)^{i}\left(\begin{array}{c}
k \\
i
\end{array}\right) f\left(x+\frac{h}{2}[k-2 i]\right) .
$$


Moreover, we consider the error of best weighted approximation of the function $f$ given by

$$
E_{m}(f)_{w_{p}, \infty}:=\inf _{P \in \mathbb{P}_{m}}\left\|(f-P) w_{p}\right\|_{\infty}
$$

where $\mathbb{P}_{m}$ denotes the set of all algebraic polynomials of degree at most $m$. Then 11, p. 185]

$$
E_{m}(f)_{w_{p}, \infty} \leq \mathcal{C} \int_{0}^{\frac{1}{\sqrt{m}}} \frac{\Omega^{k}(f, t)_{w_{p}, \infty}}{t} d t
$$

and

$$
\Omega^{k}(f, t)_{w_{p}, \infty} \leq \mathcal{C} t^{k} \sum_{1 \leq i \leq \frac{1}{t^{2}}} i^{\frac{k}{2}-1} E_{i}(f)_{w_{p}, \infty}
$$

hold. The first inequality is a weaker version of the Jackson theorem; the second one is a Stechkin-type inequality. In particular, if $f \in W_{r}^{\infty}$, then we have

$$
E_{m}(f)_{w_{p}, \infty} \leq \mathcal{C} \frac{\left\|f^{(r)} w_{p}\right\|_{\infty}}{(\sqrt{m})^{r}}
$$

In the sequel we will denote by $\tilde{W}_{r}^{\infty}$ the space of the functions $f$ belonging to $W_{r}^{\infty}, r \geq 0$, such that, for some $x_{0}>0$, we have

$$
\left|f^{(r)}(x) w_{p}(x)\right| \leq \mathcal{C} \frac{\left\|f^{(r)} w_{p}\right\|_{\infty}}{|x|^{1+\lambda}}, \quad \lambda>1, \quad|x|>x_{0} .
$$

Such functions frequently appear in the applications and sometimes $|x|^{1+\lambda}$ is replaced by $e^{|x|}$ (see for example [3], [16]).

Orthonormal polynomials. Since we have to recall some properties of the Hermite polynomials, we assume $w_{p}(x)=w(x):=e^{-x^{2}}$ (i.e., $p=1$ ), but we remark that the same properties can be easily extended by dilation in the more general case $w_{p}(x)=e^{-(p x)^{2}}$, with $p \neq 0$. Let $\left\{p_{m}(w)\right\}_{m \in \mathbb{N}}$ be the sequence of the Hermite orthonormal polynomials with positive leading coefficient, i.e.,

$$
\begin{gathered}
p_{m}(w)=\gamma_{m}(w) x^{m}+\ldots, \quad \gamma_{m}(w)>0, \\
\int_{\mathbb{R}} p_{m}(w, x) p_{n}(w, x) w(x) d x=\delta_{m, n} .
\end{gathered}
$$

The zeros $x_{k}:=x_{m, k}(w), k=1, \ldots, m$, of $p_{m}(w)$ satisfy

$$
-\sqrt{2 m}=x_{0}<x_{1}<\ldots<x_{m}<x_{m+1}=\sqrt{2 m} \text {. }
$$

Furthermore, setting $\phi_{m}(x):=\left(\sqrt{2 m-x^{2}+(2 m)^{\frac{1}{3}}}\right)^{-1}$, then

$$
\Delta x_{k}=x_{k+1}-x_{k} \sim \phi_{m}\left(x_{k}\right) \sim \frac{1}{\sqrt{2 m-x_{k}^{2}}}, \quad k=0, \ldots, m
$$

and

$$
\phi_{m}\left(x_{k}\right) \sim \phi_{m}(\xi) \sim \phi_{m}\left(x_{k+1}\right), \quad \xi \in\left[x_{k}, x_{k+1}\right], \quad k=0, \ldots, m,
$$

hold uniformly with respect to $m$ and $k$. The previous relations can be easily deduced from [17] and [39]. The following lemma is new and will be useful in the sequel. 
Lemma 2.1. Let $x_{m+1, k}, k=1, \ldots, m+1$, be the zeros of $p_{m+1}(w)$. Then

$$
\frac{1}{x_{m+1, k+1}-x_{m, k}} \leq \mathcal{C} m^{\frac{3}{2}} \phi_{m}^{2}\left(x_{m+1, k+1}\right), \quad k=1, \ldots, m,
$$

holds uniformly with respect to $m$ and $k$.

Consequently, if $x_{m+1, k+1}, x_{m, k} \in(-\theta \sqrt{2 m}, \theta \sqrt{2 m})$, with $0<\theta<1$ fixed, then

$$
\frac{1}{x_{m+1, k+1}-x_{m, k}} \leq \mathcal{C} \sqrt{m}
$$

The Christoffel functions $\lambda_{m}(w, x)$ are defined by

$$
\lambda_{m}(w, x)=\left[\sum_{i=0}^{m-1} p_{i}^{2}(w, x)\right]^{-1}
$$

and $\lambda_{k}(w):=\lambda_{m, k}(w)=\lambda_{m}\left(w, x_{k}\right), k=1, \ldots, m$, are the Christoffel numbers. In the sequel we will use the well-known estimates [17]:

$$
\lambda_{m}(w, x) \sim e^{-x^{2}} \phi_{m}(x)
$$

and

$$
\lambda_{k}(w) \sim \frac{e^{-x_{k}^{2}}}{\sqrt{2 m-x_{k}^{2}+(2 m)^{\frac{1}{3}}}} \sim \Delta x_{k} e^{-x_{k}^{2}},
$$

where the constants in " $\sim$ " are independent of $m, x$ and $k$.

Lagrange interpolation. Let us denote by $L_{m+2}(w, f), f \in W_{0}^{\infty}$, the Lagrange polynomial based on the $m+2$ knots

$$
-\sqrt{2 m}=x_{0}<x_{1}<\ldots<x_{m}<x_{m+1}=\sqrt{2 m},
$$

where $x_{k}:=x_{m, k}(w), k=1, \ldots, m$, are the zeros of $p_{m}(w)$. For this interpolation process we can state the following

Theorem 2.2. For all $f \in W_{0}^{\infty}$, the estimate

$$
\left\|\left[f-L_{m+2}(w, f)\right] \sqrt{w}\right\|_{\infty} \leq \mathcal{C} E_{m+1}(f)_{\sqrt{w}, \infty} \log m
$$

holds, where $\mathcal{C}$ is a positive constant independent of $m$ and $f$.

Theorem 2.2 is a simple but useful modification of a previous result by J. Szabados [39]. In Section 4 we will give the proof of this theorem not only for completeness, but also because we can deduce a slightly more general estimate. In fact, for $w_{p}(x):=e^{-(p x)^{2}}, p \neq 0$, we have $\left\{p_{m}\left(w_{p}, x\right)\right\}_{m}=\left\{\sqrt{p} p_{m}(w, p x)\right\}_{m}$ and

$$
p_{m}\left(w_{p}, z_{k}\right)=0 \Leftrightarrow z_{k}=\frac{x_{k}}{p}, \quad k=1, \ldots, m .
$$

If we denote by $L_{m+2}\left(w_{p}, f\right)$ the Lagrange polynomial based on the knots $\left\{x_{k} / p\right\}_{k=0,1, \ldots, m+1}$, then, from Theorem 2.2, we can easily deduce the estimate

$$
\left\|\left[f-L_{m+2}\left(w_{p}, f\right)\right] \sqrt{w_{p}}\right\|_{\infty} \leq \mathcal{C} E_{m+1}(f)_{\sqrt{w_{p}}, \infty} \log m
$$

where $\mathcal{C}$ is a positive constant independent of $m$ and $f$. 
Finally, we want to state a simple proposition that will be useful in the sequel. Let $f \in W_{r}^{\infty}, r>0$, and define $f_{r}$ as follows

$$
f_{r}(x)= \begin{cases}T_{r-1}\left(-\frac{\sqrt{2 m}}{p}\right), & x \leq-\frac{\sqrt{2 m}}{p}, \\ f(x), & |x|<\frac{\sqrt{2 m}}{p}, \\ \tilde{T}_{r-1}\left(\frac{\sqrt{2 m}}{p}\right), & x \geq \frac{\sqrt{2 m}}{p},\end{cases}
$$

where $T_{r-1}$ and $\tilde{T}_{r-1}$ are the Taylor polynomials of $f$ of degree $r-1$ with starting points $-\frac{\sqrt{2 m}}{p}$ and $\frac{\sqrt{2 m}}{p}$, respectively.

Obviously $f_{r} \in W_{r}^{\infty}$ and the next proposition holds.

Proposition 2.3. For all $f \in \tilde{W}_{r}^{\infty}$ and $\sqrt{2 m}>x_{0}$, we have

$$
\sup _{|t| \leq \frac{\sqrt{2 m}}{p}}\left|\int_{\mathbb{R}} \frac{f(x)-f_{r}(x)}{x-t} w_{p}(x) d x\right| \leq \mathcal{C} \frac{\left\|f^{(r)} w_{p}\right\|_{\infty}}{(\sqrt{m})^{r+\lambda-1}}
$$

where $\lambda>1$ and $\mathcal{C}$ is a positive constant independent of $m, f$ and $t$.

If the decay condition (2.7) in the definition of $\tilde{W}_{r}^{\infty}$ is replaced by the following

$$
\left|f^{(r)}(x) w_{p}(x)\right| \leq \mathcal{C} \frac{\left\|f^{(r)} w_{p}\right\|_{\infty}}{e^{|x|}}, \quad|x|>x_{0},
$$

then the quantity $(\sqrt{m})^{r}$ on the right-hand side of (2.16) is replaced by $o\left(e^{-\sqrt{m}}\right)$.

Now we are able to establish the main results of this paper.

\section{Main Results}

Pointwise approximation of $H(f w, t)$. In this subsection we introduce some simple procedures useful for the numerical computation of $H(f w, t), w(x)=e^{-x^{2}}$. Following an idea in [13, let us consider the following identity

$$
H(f w, t)=f(t) \int_{\mathbb{R}} \frac{e^{-x^{2}}}{x-t} d x+\int_{\mathbb{R}} \frac{f(x)-f(t)}{x-t} e^{-x^{2}} d x .
$$

To compute the first integral on the right-hand side, the reader can consult [31]. For the second one we apply the Gauss quadrature rule

$$
\int_{\mathbb{R}} f(x) e^{-x^{2}} d x=\sum_{k=1}^{m} \lambda_{k}(w) f\left(x_{k}\right), \quad \forall f \in \mathbb{P}_{2 m-1},
$$

where $x_{k}, k=1, \ldots, m$, are the Hermite zeros and $\lambda_{k}(w), k=1, \ldots, m$, are the Christoffel numbers. After some simple computations we obtain the following formula

$$
\begin{aligned}
H(f w, t) & =f(t)\left[\int_{\mathbb{R}} \frac{e^{-x^{2}}}{x-t} d x-\sum_{k=1}^{m} \frac{\lambda_{k}(w)}{x_{k}-t}\right]+\sum_{k=1}^{m} \lambda_{k}(w) \frac{f\left(x_{k}\right)}{x_{k}-t}+r_{m}(f, t) \\
& :=\Phi_{m}(f w, t)+r_{m}(f, t),
\end{aligned}
$$

where $t \neq x_{k}, k=1, \ldots, m$, and $r_{m}(f)$ is the remainder term. $\Phi_{m}(f w)$ is a Gausstype quadrature rule and it has degree of exactness $2 m$ (i.e., $r_{m}(f, t)=0, \forall f \in$ $\left.\mathbb{P}_{2 m}\right)$. Unfortunately the relation $t \neq x_{k}, k=1, \ldots, m$, is not always verified. But even when $t \neq x_{k}, k=1, \ldots, m$, the point $t$ could be too close to one of the 
Hermite zeros and this produces numerical instability. On the other hand the only term in (3.3) causing numerical instability is

$$
\frac{f\left(x_{d}\right)-f(t)}{x_{d}-t} \lambda_{d}(w)
$$

where $x_{d}$ is the zero closest to $t$. Following an argument in [27], we now introduce an algorithm in order to control this term.

For every fixed $t$, choose $m_{0}=m_{0}(t) \in \mathbb{N}$ such that, for $m \geq m_{0}$, we have $x_{m, d} \leq$ $t \leq x_{m, d+1}$ for some $d \in\{1,2, \ldots, m-1\}$.

Moreover, because of the interlacing properties of the zeros $x_{m+1, k}, k=$ $1, \ldots, m+1$, of $p_{m+1}(w)$, we have

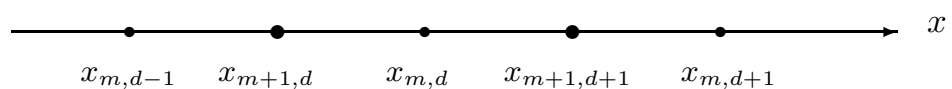

Thus, two cases are possible:

In case (a),

$$
\text { (a) } x_{m+1, d+1} \leq t \leq x_{m, d+1} \quad \text { or } \quad \text { (b) } x_{m, d} \leq t \leq x_{m+1, d+1} \text {. }
$$

if $t<\frac{x_{m+1, d+1}+x_{m, d+1}}{2}$, then we use the quadrature rule $\Phi_{m}(f w)$;

if $t \geq \frac{x_{m+1, d+1}+x_{m, d+1}}{2}$, then we use the quadrature rule $\Phi_{m+1}(f w)$.

Similarly in case (b). Thus, for every fixed $t$, we have defined the numerical sequence $\left\{\Phi_{m^{*}}(f w, t)\right\}, m^{*} \in\{m, m+1\}$.

Moreover the algorithm for the choice of $m^{*}$ is based on Lemma 2.1 and it assures us that the knot of $\Phi_{m^{*}}(f w)$ closest to $t$ is sufficiently far from $t$. In fact, for $m$ sufficiently large, $|t| \leq \theta \sqrt{2 m}$, with $0<\theta<1$ fixed, we have

$$
\left|x_{m^{*}, d}-t\right| \geq \frac{\mathcal{C}}{\sqrt{m}}
$$

The next theorem deals with the convergence of the numerical sequence $\left\{\Phi_{m^{*}}(f w, t)\right\}$. We assume $f \in \tilde{W}_{r}^{\infty}, r>0$, but, with minor effort, we can estimate the error for different classes of functions.

Theorem 3.1. Let $t$ be fixed on $\mathbb{R}$ and let $m$ be such that $|t| \leq \theta \sqrt{2 m}$, with $0<$ $\theta<1$. Then, for all $f \in \tilde{W}_{r}^{\infty}, r>0$, we have

$$
\left|r_{m^{*}}(f, t)\right|:=\left|H(f w, t)-\Phi_{m^{*}}(f w, t)\right| \leq \mathcal{C} \frac{\left\|f^{(r)} w\right\|_{\infty}}{(\sqrt{m})^{r}} \log m,
$$

where $\mathcal{C}$ is a positive constant independent of $m$ and $f$.

Theorem 3.1 shows also that, for any fixed $t$ on $\mathbb{R}$, the numerical sequence $\left\{\Phi_{m^{*}}(f w, t)\right\}$ converges to $H(f w, t)$. In particular, if $t=0$, then it is easy to see that $\Phi_{m^{*}}(f w, 0)=\Phi_{2 m}(f w, 0)$. More generally, if for any $m$ we choose

$$
t=t_{k}=\frac{x_{k}+x_{k+1}}{2}, \quad k=1, \ldots, m-1,
$$

then we can evaluate $H\left(f w, t_{k}\right)$ with the required accuracy and we can reconstruct the function $H(f w, t)$, in the interval $(-\theta \sqrt{2 m}, \theta \sqrt{2 m})$, by means of suitable interpolating splines. 
Numerical considerations. Theorem 3.1 holds under the condition $|t|<\theta \sqrt{2 m}$, i.e., since $t$ is fixed, for $m>\frac{1}{2}\left(\frac{t}{\theta}\right)^{2}$. Thus, when $t$ is "large" (but not too large), then the computation of the Christoffel numbers and of the function $f$ on the zeros of $p_{m}(w)$ is too expensive and sometimes impossible (for example, if $t=100$, then we need $m>5000)$. When this happens, i.e., $t$ is "large", we propose to approximate $H(f w, t)$ by the formula

$$
H(f w, t)=\sum_{k=1}^{m} \frac{f\left(x_{k}\right)}{x_{k}-t} \lambda_{k}(w)+\rho_{m}(f, t),
$$

where $1 \leq x_{m} \leq t-1$ and $\rho_{m}(f, t)$ is the remainder term.

Moreover, in [10] it has been proved that, for all $f \in W_{r}^{\infty}$, we have

$$
\left|\rho_{m}(f, t)\right| \leq \frac{\mathcal{C}}{(\sqrt{m})^{r}} \max _{0 \leq k \leq r}\left\|f^{(k)} w\right\|_{\infty} .
$$

So, using (3.3) together with (3.5), we obtain an efficient procedure for the computation of $H(f w, t)$, for different domains of $t$. Obviously (3.3) and (3.5) can also be used after making suitable transformations in order to regularise the density function $f$.

Uniform approximation of $H(f w, t)$. In order to construct a uniform approximation of $H(f w)$ by means of algebraic polynomials, we recall that, at the present time, the only polynomial processes convergent in $W_{0}^{\infty}$ are the de la Vallée Poussin means [30]. Obviously, the computation of the Fourier coefficients does not allow us to use such polynomials. Then, taking into account the estimate (2.14), with $p=\sqrt{2}$, it seems natural to replace $f$ by $L_{m+2}\left(w^{2}, f\right)$ in $H(f w, t)$ and to define the sequence $\left\{H_{m}(f w, t)\right\}_{m \in \mathbb{N}}$, where

$$
H_{m}(f w, t)=H\left(L_{m+2}\left(w^{2}, f\right) w, t\right) .
$$

We can also write $H_{m}(f w, t)$ as

$$
\begin{aligned}
H_{m}(f w, t)= & {\left[\frac{f(\sqrt{m})}{2 \sqrt{m} p_{m}\left(w^{2}, \sqrt{m}\right)}-\frac{f(-\sqrt{m})}{2 \sqrt{m} p_{m}\left(w^{2},-\sqrt{m}\right)}\right] A_{m} } \\
& +\left[\frac{f(\sqrt{m})(\sqrt{m}+t)}{2 \sqrt{m} p_{m}\left(w^{2}, \sqrt{m}\right)}+\frac{f(-\sqrt{m})(\sqrt{m}-t)}{2 \sqrt{m} p_{m}\left(w^{2},-\sqrt{m}\right)}\right] q_{m}(w, t) \\
& +\sum_{k=1}^{m} \frac{f\left(x_{k}\right) \lambda_{k}\left(w^{2}\right)}{\left(x_{k}^{2}-m\right)} \sum_{i=0}^{m-1} p_{i}\left(w^{2}, x_{k}\right)\left[t A_{i}+B_{i}\right] \\
& +\sum_{k=1}^{m} \frac{f\left(x_{k}\right) \lambda_{k}\left(w^{2}\right)\left(t^{2}-m\right)}{\left(x_{k}^{2}-m\right)} \sum_{i=0}^{m-1} p_{i}\left(w^{2}, x_{k}\right) q_{i}(w, t),
\end{aligned}
$$

where $\quad A_{i}=\int_{\mathbb{R}} p_{i}\left(w^{2}, x\right) w(x) d x, \quad q_{i}(w, t)=H\left(p_{i}\left(w^{2}\right) w, t\right), \quad i=0, \ldots, m, \quad$ and $B_{i}=\int_{\mathbb{R}} x p_{i}\left(w^{2}, x\right) w(x) d x, i=0, \ldots, m-1$. The coefficients $A_{i}, i=0, \ldots, m$, and $B_{i}, i=0, \ldots, m-1$, can be computed by using (3.2). To compute $q_{i}(w, t), i=$ $0, \ldots, m$, we can use the following recurrence relation:

$$
\left\{\begin{array}{l}
q_{0}(w, t)=\frac{2^{\frac{1}{4}}}{h_{0}} \int_{\mathbb{R}} \frac{e^{-x^{2}}}{x-t} d x, \quad q_{1}(w, t)=\frac{2^{\frac{7}{4}}}{h_{1}} \sqrt{\pi}+2 \sqrt{2} t \frac{h_{0}}{h_{1}} q_{0}(w, t), \\
q_{i}(w, t)=2 \sqrt{2} t \frac{h_{i-1}}{h_{i}} q_{i-1}(w, t)-2(i-1) \frac{h_{i-2}}{h_{i}} q_{i-2}(w, t), \quad i=2, \ldots, m
\end{array}\right.
$$


where $h_{i}=\left(\sqrt{\sqrt{\pi} 2^{i} i !}\right)^{-1}, i=0,1, \ldots, m$. To compute $q_{0}(w, t)$ some good routines are available (see for instance [31]).

The convergence and the estimate of the approximation error $e_{m}(f, t):=$ $H(f w, t)-H_{m}(f w, t)$ are shown by the following

Theorem 3.2. For all $f \in \tilde{W}_{r}^{\infty}, r>0$, we have

$$
\sup _{|t| \leq \sqrt{2 m}}\left|e_{m}(f, t)\right| \leq \mathcal{C} \frac{\left\|f^{(r)} w\right\|_{\infty}}{(\sqrt{m})^{r}} \log ^{2} m
$$

where $\sqrt{2 m}>x_{0}$ and $\mathcal{C}$ is a positive constant independent of $m$ and $f$.

Theorem [3.2 shows also that $H_{m}(f w, t)$ is a stable approximation of $H(f w, t)$ (except for a $\log ^{2} m$ factor) and, even if it has a greater computational cost than the previous procedure, it can be used for the computation of $H(f w, t)$.

On the other hand $H_{m}(f w, t)$ can prove to be useful for another kind of problem. In fact, as already mentioned in the Introduction, sometimes $H(f w, t)$ appears as the main part of the singular integral equations and, if we use a collocation method, then $H(f w, t)$ can be replaced by $H_{m}(f w, t)$. In this case we have to compute $f\left(x_{i}\right)$ $\left(\left|x_{i}\right| \leq \sqrt{m}\right)$ by a linear system. Thus, Theorem 3.2 is essential for the stability and the well-conditioning of the matrix of the resulting linear system.

\section{Proofs}

Proof of Lemma 2.1. Let $Q_{2 m+1}(x)=p_{m+1}(w, x) p_{m}(w, x)$. Since the zeros of $p_{m}(w)$ interlace with the zeros of $p_{m+1}(w)$, then $Q_{2 m+1}^{\prime}\left(x_{m+1, k}\right)>0$, $Q_{2 m+1}^{\prime}\left(x_{m, k}\right)<0$ and

$$
0<Q_{2 m+1}^{\prime}\left(x_{m+1, k+1}\right)-Q_{2 m+1}^{\prime}\left(x_{m, k}\right)=\left(x_{m+1, k+1}-x_{m, k}\right) Q_{2 m+1}^{\prime \prime}\left(\xi_{k}\right),
$$

where $x_{m, k}<\xi_{k}<x_{m+1, k+1}$. Consequently

$$
\frac{1}{x_{m+1, k+1}-x_{m, k}}<\frac{\left|Q_{2 m+1}^{\prime \prime}\left(\xi_{k}\right)\right|}{Q_{2 m+1}^{\prime}\left(x_{m+1, k+1}\right)} .
$$

It is easily seen that

$$
Q_{2 m+1}^{\prime \prime}\left(\xi_{k}\right) \leq \mathcal{C} \sqrt{m(m+1)} p_{m}^{2}\left(w, \xi_{k}\right) .
$$

On the other hand, by (2.12) and $\frac{\gamma_{m+1}}{\gamma_{m}}=\frac{1}{\sqrt{2(m+1)}} \sim \frac{1}{\sqrt{m}}$, we get

$$
\begin{aligned}
Q_{2 m+1}^{\prime}\left(x_{m+1, k+1}\right) & =\frac{\gamma_{m+1}}{\gamma_{m}} \frac{1}{\lambda_{m+1, k+1}(w)} \\
& \geq \frac{1}{\sqrt{2(m+1)}} \frac{\sqrt{2(m+1)-x_{m+1, k+1}^{2}}}{e^{-x_{m+1, k+1}^{2}}}
\end{aligned}
$$

Thus

$$
\frac{1}{x_{m+1, k+1}-x_{m, k}}<\mathcal{C} m \sqrt{m+1} \frac{e^{-x_{m+1, k+1}^{2}}}{\sqrt{2(m+1)-x_{m+1, k+1}^{2}}} p_{m}^{2}\left(w, \xi_{k}\right)
$$


Moreover, taking into account (4.4), we obtain

$$
\frac{1}{x_{m+1, k+1}-x_{m, k}} \leq \mathcal{C} \frac{m^{3 / 2}}{2 m-x_{m+1, k+1}^{2}},
$$

which proves Lemma 2.1 .

We recall some equivalences that are useful in the sequel:

$$
\begin{gathered}
\left|\sqrt{w\left(x_{k}\right)} p_{m}^{\prime}\left(w, x_{k}\right)\right| \sim \sqrt[4]{2 m-x_{k}^{2}+m^{\frac{1}{3}}}, \quad k=1, \ldots, m, \\
\max _{x \in \mathbb{R}}\left|\sqrt{w(x)} p_{m}(w, x) \sqrt[4]{2 m-x^{2}+m^{\frac{1}{3}}}\right| \sim 1
\end{gathered}
$$

and

$$
w(x) p_{m}^{2}(w, x) \sqrt{2 m-x^{2}+m^{\frac{1}{3}}} \sim\left(\frac{x-x_{d}}{x_{d}-x_{d \pm 1}}\right)^{2},
$$

$|x| \leq \sqrt{2 m}$. The first two equivalences follow by $[8$, Lemma 4.2 (4.6) and (4.11), p. $266]$; the third is equivalent to

$$
\frac{w(x)}{w\left(x_{d}\right)} l_{m, d}^{2}(x) \sim 1
$$

where $x_{d}$ is the zero of $p_{m}(w)$ closest to $t$, i.e.,

$$
\left|x_{d}-t\right|=\min _{k=1, \ldots, m}\left|t-x_{k}\right|
$$

and

$$
l_{m, d}(x)=\frac{p_{m}(w, x)}{p_{m}^{\prime}\left(w, x_{d}\right)\left(x-x_{d}\right)} .
$$

Proof of Theorem 2.2. Now, for $|x| \leq \sqrt{2 m}$, we can write

$$
\begin{aligned}
L_{m+2}(w, f ; x) \sqrt{w(x)}= & \frac{(\sqrt{2 m}-x) \sqrt{w(x)} p_{m}(w, x) f(-\sqrt{2 m})}{2 \sqrt{2 m} p_{m}(w,-\sqrt{2 m})} \\
& +\sqrt{w(x)} \sum_{k=1}^{m} \frac{\left(2 m-x^{2}\right) l_{m, k}(x) f\left(x_{k}\right)}{\left(2 m-x_{k}^{2}\right)} \\
& +\frac{(\sqrt{2 m}+x) \sqrt{w(x)} p_{m}(w, x) f(\sqrt{2 m})}{2 \sqrt{2 m} p_{m}(w, \sqrt{2 m})} \\
:= & A+B+C .
\end{aligned}
$$

We recall that, from the Rahmanov-Mhaskar-Saff identity, we have

$$
\max _{x \in \mathbb{R}}\left|L_{m+2}(w, f ; x) \sqrt{w(x)}\right|=\max _{x \in[-\sqrt{2 m}, \sqrt{2 m}]}\left|L_{m+2}(w, f ; x) \sqrt{w(x)}\right|
$$

and from 4.3) we get

$$
\left|p_{m}(w, x) \sqrt{w(x)}\right| \leq \mathcal{C} m^{-\frac{1}{12}} .
$$

Moreover, from (4.4) with $x= \pm \sqrt{2 m}$ (i.e., $x_{d}=x_{m}$ or $x_{1}$ ), we can also deduce

$$
\left|p_{m}(w, \pm \sqrt{2 m}) \sqrt{w( \pm \sqrt{2 m})}\right| \geq \mathcal{C} m^{-\frac{1}{12}} .
$$


Therefore

$$
\begin{aligned}
|A|+|C| \leq\|f \sqrt{w}\|_{\infty}\left\{\frac{\left\|\sqrt{w} p_{m}(w)\right\|_{\infty}}{\left|\sqrt{2 m} \sqrt{w(-\sqrt{2 m})} p_{m}(w,-\sqrt{2 m})\right|}\right. \\
\left.\quad+\frac{\left\|\sqrt{w} p_{m}(w)\right\|_{\infty}}{\left|\sqrt{2 m} \sqrt{w(\sqrt{2 m})} p_{m}(w, \sqrt{2 m})\right|}\right\} \\
\leq \mathcal{C}\|f \sqrt{w}\|_{\infty} .
\end{aligned}
$$

Now we estimate $B$. By (4.3), we have

$$
\left|\sqrt[4]{2 m-x^{2}} \sqrt{w(x)} p_{m}(w, x)\right| \leq \mathcal{C}
$$

and by (4.2) and (2.8) we get

$$
\left|\sqrt[4]{2 m-x_{k}^{2}} \sqrt{w\left(x_{k}\right)} p_{m}^{\prime}\left(w, x_{k}\right)\right| \leq \mathcal{C}\left|x_{k}-x_{k-1}\right|^{-1}:=\mathcal{C} \Delta^{-1} x_{k} .
$$

Recalling that $\left|l_{m, d}(x)\right| \sim 1$ and $\frac{2 m-x^{2}}{2 m-x_{d}^{2}} \sim 1$, we deduce

$$
|B| \leq \mathcal{C}\|f \sqrt{w}\|_{\infty}\left[1+\sum_{\substack{k=1 \\ k \neq d}}^{m}\left(\frac{2 m-x^{2}}{2 m-x_{k}^{2}}\right)^{\frac{3}{4}} \frac{\Delta x_{k}}{\left|x-x_{k}\right|}\right] .
$$

We have only to prove that the sum on the right-hand side of (4.7) is dominated by $\log m$. But it has been proved in [39].

Finally, substituting (4.6) and (4.7) into (4.5), for $|x| \leq \sqrt{2 m}$, we obtain

$$
\left|L_{m+2}(w, f ; x) \sqrt{w(x)}\right| \leq \mathcal{C}\|f \sqrt{w}\|_{\infty} \log m
$$

and, from the Rahmanov-Mhaskar-Saff identity, it follows that

$$
\left\|L_{m+2}(w, f) \sqrt{w}\right\|_{\infty} \leq \mathcal{C}\|f \sqrt{w}\|_{\infty} \log m, \quad \mathcal{C} \neq \mathcal{C}(m, f) .
$$

Then the inequality (2.13) easily follows.

Proof of Proposition [2.3. By the definition of $f_{r}$, we have

$$
\begin{aligned}
\left|\int_{\mathbb{R}} \frac{f(x)-f_{r}(x)}{x-t} w_{p}(x) d x\right| \leq & \left\{\int_{-\infty}^{-\frac{\sqrt{2 m}}{p}} \frac{\left|f(x)-f_{r}(x)\right|}{t-x} w_{p}(x) d x\right. \\
& \left.+\int_{\frac{\sqrt{2 m}}{p}}^{\infty} \frac{\left|f(x)-f_{r}(x)\right|}{x-t} w_{p}(x) d x\right\} \\
= & I_{1}+I_{2} .
\end{aligned}
$$

Taking into account that

$$
\left|f(x)-f_{r}(x)\right| \leq \int_{\frac{\sqrt{2 m}}{p}}^{x} \frac{(x-u)^{r-1}}{(r-1) !}\left|f^{(r)}(u)\right| d u,
$$


we get

$$
\begin{aligned}
I_{2} & \leq \mathcal{C} \int_{\frac{\sqrt{2 m}}{p}}^{\infty}\left|f^{(r)}(u)\right| \int_{u}^{\infty} w_{p}(x) \frac{(x-u)^{r-1}}{x-t} d x d u \\
& \leq \mathcal{C} \int_{\frac{\sqrt{2 m}}{p}}^{\infty}\left|f^{(r)}(u)\right| \int_{u}^{\infty} w_{p}(x)(x-u)^{r-2} d x d u \\
& \leq \mathcal{C} \int_{\frac{\sqrt{2 m}}{p}}^{\infty} \frac{\left|f^{(r)}(u)\right|}{u^{r-1}} w_{p}(u) d u \\
& \leq \frac{\mathcal{C}}{(\sqrt{2 m})^{r+\lambda-1}}\left\|f^{(r)} w_{p}\right\|_{\infty} .
\end{aligned}
$$

Analogously, we can prove that

$$
I_{1} \leq \frac{\mathcal{C}}{(\sqrt{2 m})^{r+\lambda-1}}\left\|f^{(r)} w_{p}\right\|_{\infty} .
$$

Substituting (4.10) and (4.11) into (4.9), we get (2.16).

In order to prove Theorems 3.1 and 3.2 we need the following lemmas.

Lemma 4.1. If $-a<-a^{\prime}<t<a^{\prime}<a, a>0$, we have

$$
\int_{-a}^{a} \frac{f(x)}{x-t} w(x) d x \leq \mathcal{C}\left\{\|f w\|_{[-a, a]} \log \frac{a}{a-a^{\prime}}+\int_{0}^{1} \frac{\Omega(f, u)_{w, \infty}}{u} d u\right\}
$$

where $\mathcal{C}$ is a positive constant independent of $f, a$ and $t$. Obviously we assume that the integral on the right-hand side exists.

Proof. Letting $\varepsilon:=\frac{\left(a-a^{\prime}\right)}{2}$, we use the following decomposition

$$
\begin{aligned}
\int_{-a}^{a} \frac{f(x)}{x-t} w(x) d x & =\left\{\int_{-a}^{t-\varepsilon}+\int_{t-\varepsilon}^{t+\varepsilon}+\int_{t+\varepsilon}^{a}\right\} \frac{f(x)}{x-t} w(x) d x \\
& :=I_{1}+I_{2}+I_{3}
\end{aligned}
$$

We have

$$
\left|I_{1}\right| \leq \int_{-a}^{t-\varepsilon} \frac{|f(x) w(x)|}{t-x} d x \leq\|f w\|_{[-a, t-\varepsilon]} \log \frac{t+a}{\varepsilon} \leq\|f w\|_{[-a, a]} \log \frac{2\left(a+a^{\prime}\right)}{a-a^{\prime}} .
$$

Analogously

$$
\left|I_{3}\right| \leq\|f w\|_{[-a, a]} \log \frac{2\left(a+a^{\prime}\right)}{a-a^{\prime}}
$$

About $I_{2}$, letting $G(x):=f(x) e^{-x^{2}}$, we can write

$$
\left|I_{2}\right|=\left|\int_{0}^{2 \varepsilon} \frac{G\left(t+\frac{u}{2}\right)-G\left(t-\frac{u}{2}\right)}{u} d u\right| \leq\left\{\int_{0}^{1}+\int_{1}^{2 \varepsilon}\right\}\left|\Delta_{\frac{u}{2}} G(t)\right| \frac{d u}{u} .
$$


If $2 \varepsilon<1$, then we let the second integral on the right-hand side equal zero. Applying the definition (2.3), we obtain

$$
\begin{aligned}
\left|I_{2}\right| \leq & \int_{0}^{1} \frac{\Omega(f, t)_{w, \infty}}{u} d u+\|f w\|_{[0,1]} \int_{0}^{1}\left|\frac{\Delta_{\frac{u}{2}} w(t)}{u w\left(t-\frac{u}{2}\right)}\right| d u \\
& +\mathcal{C}\|f w\|_{[-a, a]} \\
\leq & \int_{0}^{1} \frac{\Omega(f, t)_{w, \infty}}{u} d u+\mathcal{C}\|f w\|_{[-a, a]} .
\end{aligned}
$$

Finally, combining (4.14), (4.16) and (4.15) with (4.13), we deduce (4.12).

Lemma 4.2. For any $f \in W_{r}^{\infty}$ and for $P \in \mathbb{P}_{m}$ the polynomial of best approximation of the function $f$, we have

$$
\int_{0}^{1} \frac{\Omega(f-P, u)_{w, \infty}}{u} d u \leq \frac{\mathcal{C}}{(\sqrt{m})^{r}}\left\|f^{(r)} w\right\|_{\infty} \log m
$$

where $\mathcal{C}$ is a positive constant independent of $m, f$ and $P$.

Proof. We can write

$$
\begin{aligned}
\int_{0}^{1} \frac{\Omega(f-P, u)_{w, \infty}}{u} d u & \leq\left\{\int_{0}^{\frac{1}{\sqrt{m}}}+\int_{\frac{1}{\sqrt{m}}}^{1} \frac{\Omega(f-P, u)_{w, \infty}}{u} d u\right. \\
& \leq \mathcal{C}\|[f-P] w\|_{\infty} \log m+\int_{0}^{\frac{1}{\sqrt{m}}} \frac{\Omega(f-P, u)_{w, \infty}}{u} d u .
\end{aligned}
$$

By using (2.5) and proceeding as in [24, Proof of Proposition 4.2, pp. 280-281], we get

$$
\int_{0}^{\frac{1}{\sqrt{m}}} \frac{\Omega(f-P, u)_{w, \infty}}{u} d u \leq \mathcal{C} \int_{0}^{\frac{1}{\sqrt{m}}} \frac{\Omega^{r}(f, u)_{w, \infty}}{u} d u .
$$

On the other hand by (2.4) we deduce

$$
\|(f-P) w\|_{\infty} \leq \mathcal{C} \int_{0}^{\frac{1}{\sqrt{m}}} \frac{\Omega^{r}(f, u)_{w, \infty}}{u} d u .
$$

Finally, taking into account

$$
\Omega^{r}(f, u)_{w, \infty} \leq \mathcal{C}\left\|f^{(r)} w\right\|_{\infty} u^{r},
$$

we get (4.17).

We recall that if a function $g$ is such that $g^{(i)}(x) \geq 0, i=0,1, \ldots, 2 m-1, m>1$, for $x \in\left(-\infty, x_{d}\right], d=2, \ldots, m$, then

$$
\sum_{i=1}^{d-1} \lambda_{i}(w) g\left(x_{i}\right) \leq \int_{-\infty}^{x_{d}} g(x) w(x) d x \leq \sum_{i=1}^{d} \lambda_{i}(w) g\left(x_{i}\right) .
$$

If $(-1)^{i} g^{(i)}(x) \geq 0, i=0,1, \ldots, 2 m-1, m>1$, for $x \in\left[x_{d}, \infty\right), d=1, \ldots, m-1$, then

$$
\sum_{i=d+1}^{m} \lambda_{i}(w) g\left(x_{i}\right) \leq \int_{x_{d}}^{\infty} g(x) w(x) d x \leq \sum_{i=d}^{m} \lambda_{i}(w) g\left(x_{i}\right)
$$

(see [ㅁ, Proof of Lemma 5.1 (b), pp. 271-272]). 
Letting

$$
A_{m}(t)=\int_{\mathbb{R}} \frac{e^{-x^{2}}}{x-t} d x-\sum_{\substack{k=1 \\ k \neq d}}^{m} \frac{\lambda_{k}(w)}{x_{k}-t}
$$

we can prove the following

Lemma 4.3. Let $|t| \leq \theta \sqrt{2 m}$, with $0<\theta<1$, then

$$
A_{m}(t) \leq \mathcal{C} e^{-t^{2}}
$$

and

$$
\frac{\lambda_{m^{*}, d}(w)}{\left|x_{m^{*}, d}-t\right|} \leq \mathcal{C} e^{-x_{d}^{2}} \leq \mathcal{C} e^{-t^{2}}
$$

hold, where $\mathcal{C}$ is a positive constant independent of $m$ and $f$ and $m^{*} \in\{m, m+1\}$.

Proof. Consider the case $x_{d-1}<x_{d} \leq t<x_{d+1}, d \in\{2, \ldots, m-1\}$. By using (4.19) and (4.20), we have

$$
\begin{aligned}
A_{m}(t) & \leq \frac{\lambda_{d-1}(w)}{t-x_{d-1}}+\int_{x_{d-1}}^{x_{d+1}} \frac{e^{-x^{2}}}{x-t} d x \\
& :=A+B .
\end{aligned}
$$

Since $t-x_{d-1} \geq\left(x_{d}-x_{d-1}\right)=\Delta x_{d-1}$, by (2.8) and (2.12), we deduce

$$
|A| \leq \mathcal{C} e^{-x_{d-1}^{2}} \text {. }
$$

Taking into account that $t^{2}-x_{d-1}^{2}=\left(t-x_{d-1}\right)\left(t+x_{d-1}\right) \leq \frac{2 \theta \sqrt{2 m}}{\sqrt{2 m} \sqrt{1-\theta}} \leq \mathcal{C}$, we get

$$
|A| \leq \mathcal{C} e^{-t^{2}} e^{t^{2}-x_{d-1}^{2}} \leq \mathcal{C} e^{-t^{2}} .
$$

To estimate $B$, we note that

$$
\begin{aligned}
B & =\int_{x_{d-1}}^{x_{d+1}} \frac{e^{-x^{2}}-e^{-t^{2}}}{x-t} d x+e^{-t^{2}} \int_{x_{d-1}}^{x_{d+1}} \frac{d x}{x-t} \\
& \leq 2 \int_{x_{d-1}}^{x_{d+1}} \xi_{x} e^{-\xi_{x}^{2}} d x+e^{-t^{2}} \log \frac{x_{d+1}-t}{t-x_{d-1}} \\
& \leq 2 x_{d+1} e^{-x_{d-1}^{2}}\left(x_{d+1}-x_{d-1}\right)+e^{-t^{2}} \log \frac{x_{d+1}-t}{t-x_{d-1}} \\
& \leq \mathcal{C} \sqrt{2 m} e^{-x_{d-1}^{2}}\left(t-x_{d-1}\right)+\mathcal{C} e^{-t^{2}} \\
& \leq \mathcal{C} e^{-t^{2}} .
\end{aligned}
$$

Thus, replacing (4.24) and (4.25) into (4.23), we prove (4.21).

To prove 4.22, we note that

$$
\left|x_{m^{*}, d}-t\right|>\mathcal{C}\left|x_{m^{*}+1, d+1}-x_{m^{*}, d}\right| .
$$

Thus, applying Lemma 2.1 and (2.12), we obtain

$$
\frac{\lambda_{d}(w)}{\left|x_{m^{*}, d}-t\right|} \leq \mathcal{C} e^{-x_{d}^{2}}\left(\frac{m}{2 m-x_{d}^{2}+(2 m)^{\frac{1}{3}}}\right)^{\frac{2}{3}} \leq \mathcal{C} e^{-x_{d}^{2}} \leq \mathcal{C} e^{-t^{2}} e^{t^{2}-x_{d}^{2}} \leq \mathcal{C} e^{-t^{2}},
$$

that is the thesis. 
Proof of Theorem 3.1. Let $p_{m} \in \mathbb{P}_{m}$ be the polynomial of best approximation of the function $f_{r}$. Recalling definition (2.15), we note that

$$
r_{m}(f, t)=\int_{\mathbb{R}} \frac{f(x)-f_{r}(x)}{x-t} w(x) d x+r_{m}\left(f_{r}, t\right),
$$

where

$$
\begin{aligned}
r_{m}\left(f_{r}, t\right)= & \int_{\mathbb{R}} \frac{f_{r}(x)-p_{m}(x)}{x-t} w(x) d x \\
& -\left[f_{r}(t)-p_{m}(t)\right] A_{m}(t)+\left[f_{r}(t)-p_{m}(t)\right] \frac{\lambda_{d}(w)}{x_{d}-t} \\
& -\left[f_{r}\left(x_{d}\right)-p_{m}\left(x_{d}\right)\right] \frac{\lambda_{d}(w)}{x_{d}-t}-\sum_{\substack{k=1 \\
k \neq d}}^{m} \frac{f_{r}\left(x_{k}\right)-p_{m}\left(x_{k}\right)}{x_{k}-t} \lambda_{k}(w) \\
= & I_{1}+I_{2}+I_{3}+I_{4}+I_{5} .
\end{aligned}
$$

We can write

$$
\begin{aligned}
I_{1} & =\left\{\int_{-\infty}^{-\sqrt{2 m}}+\int_{\sqrt{2 m}}^{\infty}+\int_{-\sqrt{2 m}}^{\sqrt{2 m}}\right\} \frac{f_{r}(x)-p_{m}(x)}{x-t} w(x) d x \\
& =A_{1}+A_{2}+A_{3} .
\end{aligned}
$$

Since $|t| \leq \theta \sqrt{2 m}$, then $x-t>(1-\theta) \sqrt{2 m}$. Therefore, we have

$$
\begin{aligned}
\left|A_{2}\right| & \leq \frac{1}{(1-\theta) \sqrt{2 m}} \int_{\sqrt{2 m}}^{\infty}\left|f_{r}(x)-p_{m}(x)\right| w(x) d x \\
& \leq \frac{1}{(1-\theta) \sqrt{2 m}} \int_{-\infty}^{\infty}\left|f_{r}(x)-p_{m}(x)\right| w(x) d x .
\end{aligned}
$$

Finally, by (2.6) we get

$$
\left|A_{2}\right| \leq \frac{1}{(1-\theta)(\sqrt{2 m})^{r+1}} \int_{-\sqrt{2 m}}^{\sqrt{2 m}}\left|f^{(r)}(x) w(x)\right| d x \leq \frac{\mathcal{C}}{(\sqrt{m})^{r}}\left\|f^{(r)} w\right\|_{\infty} .
$$

Analogously we can prove that

$$
\left|A_{1}\right| \leq \frac{\mathcal{C}}{(\sqrt{m})^{r}}\left\|f^{(r)} w\right\|_{\infty} .
$$

About $A_{3}$, applying Lemma 4.1 with $a=\sqrt{2 m}$ and $a^{\prime}=\theta \sqrt{2 m}$, we get

$$
\left|A_{3}\right| \leq \mathcal{C}\left\{\left\|\left(f_{r}-p_{m}\right) w\right\|_{\infty}+\int_{0}^{1} \frac{\Omega\left(f_{r}-p_{m}, u\right)_{w, \infty}}{u} d u\right\}
$$

Moreover, taking into account Lemma 4.2 and (2.6) , we get

$$
\left|A_{3}\right| \leq \frac{\mathcal{C}}{(\sqrt{m})^{r}}\left\|f^{(r)} w\right\|_{\infty} \log m
$$

Substituting (4.30), (4.29), (4.31) into (4.28), we obtain

$$
\left|I_{1}\right| \leq \frac{\mathcal{C}}{(\sqrt{m})^{r}}\left\|f^{(r)} w\right\|_{\infty} \log m .
$$

Applying Lemma 4.3 and (2.6), we obtain

$$
\left|I_{2}\right|+\left|I_{3}\right|+\left|I_{4}\right| \leq \mathcal{C}\left\|\left(f_{r}-p_{m}\right) w\right\|_{\infty} \leq \frac{\mathcal{C}}{(\sqrt{m})^{r}}\left\|f^{(r)} w\right\|_{\infty} .
$$


Moreover, taking into account (2.12) we get

$$
\left|I_{5}\right| \leq \mathcal{C}\left\|\left(f_{r}-p_{m}\right) w\right\|_{\infty} \sum_{\substack{k=1 \\ k \neq d}}^{m} \frac{\Delta x_{k}}{\left|x_{k}-t\right|} .
$$

Since it is easy to see that

$$
\sum_{\substack{k=1 \\ k \neq d}}^{m} \frac{\Delta x_{k}}{\left|x_{k}-t\right|} \leq \mathcal{C} \log m
$$

applying (2.6), we have

$$
\left|I_{5}\right| \leq \frac{\mathcal{C}}{(\sqrt{m})^{r}}\left\|f^{(r)} w\right\|_{\infty} \log m
$$

Combining (4.32), (4.33) and (4.34) with (4.27), we get

$$
r_{m}\left(f_{r}, t\right) \leq \frac{\mathcal{C}}{(\sqrt{m})^{r}}\left\|f^{(r)} w\right\|_{\infty} \log m .
$$

Finally, substituting (4.35) into (4.26) and applying Proposition [2.3], with $p=1$, we deduce (3.4).

Proof of Theorem 3.2] We can write

$$
e_{m}(f, t)=\int_{\mathbb{R}} \frac{f(x)-f_{r}(x)}{x-t} w(x) d x+e_{m}\left(f_{r}, t\right),
$$

where

$$
\begin{aligned}
e_{m}\left(f_{r}, t\right) & \\
& =\int_{\mathbb{R}} \frac{f_{r}(x)-L_{m+2}\left(w^{2}, f_{r}, x\right)}{x-t} w(x) d x \\
& =\left\{\int_{-\infty}^{-\sqrt{2 m}-1}+\int_{\sqrt{2 m}+1}^{\infty}+\int_{-\sqrt{2 m}-1}^{\sqrt{2 m}+1}\right\} \frac{f_{r}(x)-L_{m+2}\left(w^{2}, f_{r}, x\right)}{x-t} w(x) d x \\
& =A_{1}+A_{2}+A_{3} .
\end{aligned}
$$

Now, we can proceed analogously to the proof of 4.28), replacing $p_{m}$ by $L_{m+2}\left(w^{2}, f_{r}\right)$. The only difference consists in the evaluation of $A_{3}$, for which we apply Lemma 4.1 with $a=\sqrt{2 m}+1$ and $a^{\prime}=\sqrt{2 m}$. Thus, we obtain

$$
\left|e_{m}\left(f_{r}, t\right)\right| \leq \frac{\mathcal{C}}{(\sqrt{m})^{r}}\left\|f^{(r)} w\right\|_{\infty} \log m
$$

Substituting (4.37) into (4.36) and applying Proposition 2.3 with $p=1$, (3.6) follows.

\section{Numerical evaluations}

In this section we show some approximate values for the integral $H(f w, t), t \in \mathbb{R}$, obtained by using the algorithm described in Section 3.

The density functions we choose are representatives of the functional spaces (e.g., Sobolev spaces) on which we want to test our method, i.e., we do not exclude the integrals that could be better calculated otherwise.

Since in the following examples the exact values of the integrals are not known, the results on the last line of our tables are thought to be exact to the number of 
TABLE 1.

\begin{tabular}{|c|l|l|l|}
\hline \multirow{2}{*}{$m$} & \multicolumn{3}{|c|}{$\Phi_{m}$} \\
\cline { 2 - 4 } & $t=0.1$ & $t=5$ & $t=10$ \\
\hline 8 & -0.261315425408 & -0.470154 & -0.2293312798 \\
\hline 16 & -0.261315425408597 & -0.47015461500803 & -0.2293312798756 \\
\hline
\end{tabular}

TABLE 2 .

\begin{tabular}{|c|l|l|l|}
\hline \multirow{2}{*}{$m$} & \multicolumn{3}{|c|}{$H_{m}$} \\
\cline { 2 - 4 } & $t=0.1$ & $t=5$ & $t=10$ \\
\hline 8 & -0.26131 & -0.47015461 & -0.2293312798 \\
\hline 16 & -0.261315425408 & -0.4701546150080 & -0.2293312798 \\
\hline 32 & -0.261315425408597 & -0.47015461500803 & -0.229331279875 \\
\hline
\end{tabular}

TABLE 3 .

\begin{tabular}{|c|c|l|c|l|}
\hline$t$ & $m$ & $Q_{m, m}^{(1)}$ & $m$ & $g_{m}$ \\
\hline 0.1 & 32 & -0.26131542540859 & 63 & -0.26131542540859 \\
\hline 5 & 32 & -0.47015461500803 & 113 & -0.47015461500803 \\
\hline 10 & 64 & -0.22933127987563 & 163 & -0.2293312798756 \\
\hline
\end{tabular}

figures shown. Moreover, in all the tables we have reported only the digits which are correct according to these exact values.

Example 1. We want to evaluate the following integral

$$
\int_{-\infty}^{\infty} \frac{\cosh x}{x-t} e^{-x^{2}} d x
$$

Since the function $f(x)=\cosh x=\frac{e^{x}+e^{-x}}{2}$ is an analytic function with an exponential growth, we obtain very accurate results. In Table[ 1 we can see that, for different values of the parameter $t$, we need only 16 points to obtain machine precision.

In Table 2 we show the corresponding results obtained by using the quadrature formula $H_{m}$.

We can note that the two formulas $\Phi_{m}$ and $H_{m}$ give results almost comparable, but $H_{m}$ has a more expensive computational cost than $\Phi_{m}$.

In Table 3 we compare our results with the ones obtained by using the quadrature rules proposed in [16] and [3]. We denote by

$$
Q_{m, m}^{(1)}=\sum_{k=-m}^{m} \frac{G\left(t+k h+\frac{h}{2}\right)}{k+\frac{h}{2}}
$$

where $h=\sqrt{\frac{2 \pi}{m}}$, the quadrature rule proposed in [3], and by

$$
g_{m}=2 \sum_{\substack{\nu=-m \\ \nu \neq e v e n}}^{m} \frac{G(t+\nu h)}{\nu}
$$

where $h=0.1$, the quadrature rule proposed in [16]. 
TABLE 4.

\begin{tabular}{|c|l|c|l|c|l|}
\hline \multicolumn{2}{|c|}{$t=0.25$} & \multicolumn{2}{c|}{$t=0.3$} & \multicolumn{2}{c|}{$t=15$} \\
\hline$m$ & $\Phi_{m}$ & $m$ & $\Phi_{m}$ & $m$ & $\Phi_{m}$ \\
\hline 9 & -480. & 9 & -470.1 & 8 & -229. \\
\hline 19 & -480.4897 & 19 & -470.1335 & 17 & -229.2247 \\
\hline 39 & -480.489721 & 38 & -470.133527 & 35 & -229.224743 \\
\hline 78 & -480.48972131 & 76 & -470.13352728 & 71 & -229.22474324 \\
\hline 157 & -480.4897213129 & 153 & -470.1335272874 & 143 & -229.2247432478 \\
\hline 314 & -480.48972131296 & 307 & -470.133527287461 & 286 & -229.224743247854 \\
\hline
\end{tabular}

TABLE $5 . \quad \alpha=2$

\begin{tabular}{|c|l|c|l|c|l|}
\hline \multicolumn{2}{|c|}{$t=-1.5$} & \multicolumn{2}{c|}{$t=5$} & \multicolumn{2}{c|}{$t=15$} \\
\hline$m$ & $\Phi_{m}$ & $m$ & $\Phi_{m}$ & $m$ & $\Phi_{m}$ \\
\hline 32 & 1.1711 & 32 & -0.3 & 32 & -0.105 \\
\hline 65 & 1.17112 & 65 & -0.32 & 64 & -0.1051 \\
\hline 131 & 1.171126 & 131 & -0.3253 & 128 & -0.1051 \\
\hline 262 & 1.1711263 & 262 & -0.32531 & 257 & -0.105179 \\
\hline
\end{tabular}

TABle $6 . \quad \alpha=3$

\begin{tabular}{|c|l|c|l|c|l|}
\hline \multicolumn{2}{|c|}{$t=0.5$} & \multicolumn{2}{c|}{$t=8$} & \multicolumn{2}{c|}{$t=18$} \\
\hline$m$ & $\Phi_{m}$ & $m$ & $\Phi_{m}$ & $m$ & $\Phi_{m}$ \\
\hline 37 & -1.77814 & 32 & -0.14 & 32 & -6.551 \\
\hline 74 & -1.77814144 & 65 & -0.14 & 64 & -6.5517 \\
\hline 149 & -1.77814144 & 131 & -0.148 & 128 & -6.55177 \\
\hline 298 & -1.7781414419 & 262 & -0.1480 & 257 & -6.55177 \\
\hline
\end{tabular}

Example 2. Now we consider the following integral

$$
\int_{-\infty}^{\infty} \frac{\left|x-\frac{1}{2}\right|^{\frac{7}{2}}\left|x-\frac{1}{3}\right|^{\frac{9}{2}}\left|x-\frac{1}{4}\right|^{\frac{9}{2}}}{x-t} e^{-x^{2}} d x .
$$

Since $f(x)=\left|x-\frac{1}{2}\right|^{\frac{7}{2}}\left|x-\frac{1}{3}\right|^{\frac{9}{2}}\left|x-\frac{1}{4}\right|^{\frac{9}{2}} \in \tilde{W}_{3}^{\infty}$, the theoretical error is $m^{-\frac{3}{2}} \log m$. The results shown in Table 4 confirm that we have to increase $m$ to reach significant digits.

Example 3. Finally we evaluate the following integral

$$
\int_{-\infty}^{\infty} \frac{e^{x^{2}}}{\left(1+x^{2}\right)^{\alpha}} \frac{e^{-x^{2}} d x}{x-t}, \quad \alpha=2,3
$$

If $\alpha=2$, then the function $f(x)=\frac{e^{x^{2}}}{\left(1+x^{2}\right)^{2}} \in \tilde{W}_{3}^{\infty}$ and the theoretical error is $m^{-\frac{3}{2}} \log m$; if $\alpha=3$, then the function $f(x)=\frac{e^{x^{2}}}{\left(1+x^{2}\right)^{3}} \in \tilde{W}_{4}^{\infty}$ and the theoretical error is $m^{-2} \log m$. In Tables 5 and 6 we can see that the numerical results agree with the theoretical ones.

All the computations were done in Double Precision Arithmetic on the Digital Ultimate Workstation 533au ${ }^{2}$. 


\section{ACKNOWLEDGMENTS}

The authors wish to thank the referees for their useful suggestions and remarks which have helped in the improvement of the paper.

\section{REFERENCES}

[1] Akhiezer N.I.: Lectures on Integral Transforms, AMS, 70 (Translations of Math. Mon.) (1988). MR 89i:44001

[2] Bari N.: Treatise on Trigonometric Series, Pergamon Press, New York (1964). MR 30:1347

[3] Bialecki B.: Sinc Quadratures for Cauchy Principal Value Integrals, Numerical Integration, Recent Developments, Software and Applications, edited by T.O. Espelid and A. Genz, NATO ASI Series, Series C: Mathematical and Physical Sciences, 357, Kluwer Academic Publishers, Dordrecht (1992). MR 93m:65030

[4] Capobianco M.R., Mastroianni G., Russo M.G.: Pointwise and Uniform Approximation of the Finite Hilbert Transform, Proceedings of ICAOR, (Romania) Cluj-Napoca, July 29-August 1, 1 (1996). MR 99b:65168

[5] Criscuolo G., Mastroianni G.: On the convergence of an interpolatory product rule for evaluating Cauchy principal value integrals, Math. Comp. 48 (1987), 725-735. MR 88m:65038

[6] Criscuolo G., Mastroianni G.: On the convergence of product formulas for the numerical evaluation of derivatives of Cauchy principal value integrals, SIAM J. Numerical Analysis 25 (1988), 713-727. MR 90b:65032

[7] Criscuolo G., Mastroianni G.: On the Uniform Convergence of Gaussian Quadrature Rules for Cauchy Principal Value Integrals, Numer. Math. 54 (1989), 445-461. MR 90h:65023

[8] Criscuolo G., Della Vecchia B., Lubinsky D.S., Mastroianni G.: Function of the Second Kind for Freud Weights and Series Expansions of Hilbert Transforms, Journal of Mathematical Analysis and Applications, 189 (1995), 256-296. MR 96b:42028

[9] Davis P.J., Rabinowitz P.: Methods of Numerical Integration, Academic Press, Inc., (1984). MR 86d:65004

[10] De Bonis M.C., Russo M.G.: Computation of the Cauchy Principal Value Integrals on the Real Line, Proceedings of the Workshop "Advanced Special Functions and Applications", eds. D. Cocolicchio, G. Dattoli and H.M. Srivastava (ARACNE, Rome, 1999).

[11] Ditzian Z., Totik V.: Moduli of Smoothness, SCM, Springer-Verlag, New York Berlin Heidelberg London Paris Tokyo, 9 (1987). MR 89h:41002

[12] Gautschi W.: A survey of Gauss-Christoffel quadrature formulae, in E.B. Christoffel, The Influence of his Work on Mathematics and Physical Sciences, Birkhäuser, Basel 1981. P.L. Butzer and F. Fehér, eds, 72-147. MR 83g:41031

[13] Hunter D.B.: Some Gauss-Type Formulae for the evaluation of Cauchy Principal Values of Integrals, Numer. Math. 19 (1972), 419-424. MR 47:7899

[14] Khavin V.P., Nikolski N.K.: Commutative harmonic analysis I, Encyclopedia of Mathematical Sciences, 15 Springer-Verlag, Berlin (1991). MR 93b:42001

[15] Kumar S.: A note on quadrature formulae for Cauchy principal value integrals, Journal of the Institute of Mathematics and Its Applications 26 (1980), 447-451. MR 82d:65027

[16] Kress V.R., Martensen E.: Anwendung der Rechteckregel auf die reelle Hilberttransformation mit unendlichem Intervall, ZAMM 50, T 61-T 64 (1970). MR 43:8240

[17] Levin A.L., Lubinsky D.S.: Christoffel Functions, Orthogonal Polynomials and Nevai's Conjecture for Freud Weights, Constr. Approx. 8 (1992), 463-535. MR 94f:42030

[18] Longman I.M.: On the numerical evaluation of Cauchy principal value integrals, Math. Comp. 12 (1958), 205-207. MR 20:6789

[19] Lubinsky D.S., Sidi A.: Convergence of product integration rules for functions with interior and endpoint singularities over bounded and unbounded intervals, Tech. Rep. No 215. Computer Science Dept., Technion, Haifa, Israel, 1981; Math. Comp. 46 (1986), no. 173, 229-245. MR 87j: 41072

[20] Mastroianni G.: On the Convergence of Product Formulas for the Evaluation of certain Two-Dimensional Cauchy Principal Value Integrals, Math. Comp. 52 (1989), 95-101. MR 90a:65049

[21] Mastroianni G., Monegato G.: Convergence of product integration rules over $(0, \infty)$ for functions with weak singularities at the origin, Mathematics of Computation 64 No 209 (1995), 237-249. MR 95c:65037 
[22] Mastroianni G., Monegato G.: Nyström interpolants based on zeros of Laguerre polynomials for some Wiener-Hopf equations, IMA Journal of Numerical Analysis (1997) 17, 621-642. MR 98j:45011

[23] Mastroianni G., Ricci P.E.: Error Estimates for a class of integral and discrete transforms, Studia Sci. Math. Hungar. 36 (2000), no. 3-4, 291-305. MR 2001h:44004

[24] Mastroianni G., Russo M.G.: Lagrange Interpolation in Weighted Besov Spaces, Const. Approx. (1999), 15, 257-289. MR 2000b:41001

[25] Monegato G.: The Numerical Evaluation of One-Dimensional Cauchy Principal Value Integrals, Computing 29 (1982), 337-354. MR 84c:65044

[26] Monegato G.: Convergence of Product Formulas for the Numerical Evaluation of certain Two-Dimensional Cauchy Principal Value Integrals, Numer. Math. 43 (1984), 161-173. MR 85h:65049

[27] Mastronardi N., Occorsio D.: Some Numerical Algorithms to evaluate Hadamard Finite-Part Integrals, J. Comput. Appl. Math. Vol. 70 (1996), 75-93. MR 97h:65019

[28] Mikhlin S.G., Prössdorf S.: Singular Integral Operator, Springer-Verlag, Berlin, Heidelberg, New York, Tokyo, (1986). MR 88e:47097

[29] Nevai P.G.: Mean Convergence of Lagrange Interpolation II, J. Approx. Theory Vol. 30 (1980), 263-276. MR 82i:41003

[30] Poiani E.L.: Mean Cesaro Summability of Laguerre and Hermite Series, Transactions AMS, 173 (1972), 1-31. MR 46:9635

[31] Poppe G.P.M., Wijers M.J.: Algorithm 680: Evaluation of the Complex Error Function, ACM Trans. Math. Software 16 (1990), pag. 47. MR 91h:65068b

[32] Sklyarov V.P.: On the convergence of Lagrange-Hermite interpolation for unbounded functions, Anal. Math, 20 (1994), 295-308 (in Russian). MR 95h:41006

[33] Sloan I.H., Smith W.E.: Properties of interpolatory product integration rules, SIAM J.Numer. Anal. 19 (1982), 427-442. MR 83e:41032

[34] Smith W.E., Sloan I.H., Opie A.H.: Product Integration Over Infinite Intervals I. Rules Based on the Zeros of Hermite Polynomials, Mathematics of Computations 40 No 162 (1983), 519535. MR 85a:65047

[35] Stenger F.: Approximations via Whittaker's cardinal function, J. Approx. Theory, 17 (1976), 222-240. MR 58:1885

[36] Stenger F.: Numerical Methods based on Whittaker cardinal or Sinc Functions, SIAM Review, 23 (1981), 165-224. MR 83g:65027

[37] Stenger F.: Numerical Methods based on Sinc and Analytic Functions, Springer-Verlag, (1993). MR 94k:65003

[38] Stenger F.: Summary of Sinc Approximation, preprint.

[39] Szabados J.: Weighted Lagrange and Hermite-Fejér interpolation on the real line, J. of Inequal. \& Appl., 1 (1997), 99-123. MR 2000h:41010

Dipartimento di Matematica, Universitì della Basilicata, C/Da Macchia Romana 85100 Potenza, ItAly

E-mail address: mdebonis@pta.unibas.it

Dipartimento di Matematica, Istituto G. Castelnuovo, Università di Roma La Sapienza, P.le Aldo Moro 2, 00185 Roma, Italy

E-mail address: dellavecchia@iamna.iam.na.cnr.it

Dipartimento di Matematica, Universitì della Basilicata, C/Da Macchia Romana 85100 Potenza, ItALY

E-mail address: mastroianni@unibas.it 